Bangladesh Journal of Anatomy January 2012, Vol. 10 No. 1 pp 37-42

\title{
Reduction of Masticatory Neurons in the Tooth Extracted and Aged Mouse
}

\author{
Mohammed Enamur Rashid ${ }^{1}$, Khaleda Akhter ${ }^{2}$, Norihiko Maeda ${ }^{3}$
}

\begin{abstract}
Background: Mastication is an integrated biological activity carried out by the combined operation of masticatory motor apparatus, sensory input from the oro-facial sensory organs and the central nervous system. It has been reported that the number of masticatory motor and sensory neurons were seriously diminished and the masseter muscles were severely atrophied in the genetically mutant osteopetrotic (op/op) mouse with no eruption of teeth and less-developed periodontal ligaments. These facts suggest that the sensory input from the periodontal mechanoreceptive afferents may be involved in the development and maintenance of the masticatory system. A long term experimental model was designed to reveal the general role of the periodontal afferent in the development and maintenance of the masticatory system in normal animal.

Materials and Method: Two groups of male ICR mice were used throughout this experiment. Each experimental lot consisted of 6 animals for assessing the number of neurons in the Trigeminal Mesencephalic Neurons (Me5) and Trigeminal Ganglion (TG) for each time frame. In the first group (control group), no tooth was extracted. In the other group, unilateral extraction of upper and lower molars were carried out on day 20 after birth. For assessing effects of tooth loss on the masticatory neurons, both intact and toothextracted mice were sacrificed on days 30,60, 90, 120, 180 and 360 after birth and the Me5 and TG neurons were traced and counted on both sides of the Central Nervous System (CNS). Horseradish peroxidase wheatgerm agglutinin (HRP-WGA) tracing method was applied to trace and count the number of masticatory neurons in the CNS.

Result: In tooth-extracted mice, severe degeneration of the ipsilateral Inferior Alveolar Nerve (IAN) was observed on as early at day 40 after birth and there was significant decrease in the number of labelled Me5 and TG neurons from 60th to 360th postnatal day on the tooth-extracted side. Labelled primary afferent terminals were also severely diminished in the Trigeminal Sensory Nuclear Complex (TSNC) on the toothextracted side. Moreover, there were significant decreases in the numbers of labeled Me5 and TG neurons in 360-day-old intact mice in comparison with those in 30-day-old intact mice.
\end{abstract}

Conclusion: Present study suggests that the decrease in the sensory input due to the tooth loss or aging causes the disorder of the development and maintenance of the masticatory system even in the normal animal.

Key Words: Aging, Masticatory neurons, Periodontal Mechano Receptors (PMR), Sensory input, Tooth extraction.

\section{Introduction:}

Mastication is an integrated biological activity that is carried out by the combined operation of masticatory motor apparatus, sensory input from the oro-facial sensory organs and the central nervous system. ${ }^{1,2,3,4,5,6,7,8,9,10,11}$ Mastication increases

1. Specialist Dentist \& Clinical Assistant Professor Assir Specialist Dental Centre, Abha, Ministry of Health, KSA.

2. Assistant Professor, Assir Specialist Dental Centre, Abha, Ministry of Health, KSA

3. Chairman, Department of Oral Growth and Developmental Biology, Faculty of Dentistry, Hiroshima University, Japan.

Correspondence to: Dr. Mohammed Enamur Rashid neuronal activities in various regions of the cerebral cortex. ${ }^{12}$ After tooth loss, the remnants of the periodontal ligaments break down and gradually disappear ${ }^{13}$ thus inducing the loss of the periodontal mechanoreceptors (PMRs) in the periodontal ligaments.

It is evident that there is impairment of spatial memory and low acetylcholine level in the brain of aged tooth-extracted rats and tooth loss might be a risk factor for senile dementia. ${ }^{14}$ Furthermore, due to reduction of the sensory input from the PMRs, severe impairment of the masticatory system is 
observed in the osteopetrotic (op/op) mouse with no erupted teeth and less-developed periodontal ligaments. ${ }^{15,16}$

Thus, it is suggested that the PMRs may be importantly involved in keeping not only the homeostasis of the masticatory system but also various brain functions. However, there has been less information on changes in the numbers of the neurons that innervate the periodontal ligaments after tooth loss or damage to the inferior alveolar nerve as well as due to age in the normal animal. From this point of view, a long-term observation is designed for the first time to make clearer how the interference in the PMRs, due to the tooth loss, and / or aging affect the masticatory system, particularly masticatory sensory neurons, even in the normal developing animal.

\section{Materials ad Methods}

This research was carried out in the Department of Oral Growth and Developmental Biology, Faculty of Dentistry, Hiroshima University, Japan between 2001 to 2003.

\section{A. Experimental animals}

Two groups of male ICR (Imprinting Control Region) mice were used throughout this experiment. Each experimental group consisted of 6 animals for assessing the number of neurons in the $\mathrm{Me} 5$ and TG for each time frame.

In the first group (control group), no tooth was extracted. Intact mice were sacrificed on days 30 , $60,90,120,180$ and 360 after birth and the Me5 and TG neurons were traced and counted on both sides of the CNS for reference. In the other group (experimental group), unilateral extraction of upper and lower molars was carried out on day 20 after birth. For assessing effects of tooth loss on the masticatory neurons, tooth-extracted mice were sacrificed on days $30,60,90,120,180$ and 360 after birth and the Me5 and TG neurons were traced and counted on both sides of the CNS.

Body weights were measured on every 5 days throughout the experiments to monitor any change in their general health and conditions, particularly the nutritional condition of the tooth-extracted mice.

B. HRP tracing methods for labeling masticatory sensory neurons and the primary afferents

To trace the masticatory neurons in the CNS, Horseradish peroxidase-wheat germ agglutinin (HRP-WGA) tracing method was applied to the mice of 30 days, 60 days, 90 day, 120 days, 180 days and 360 days after birth, with or without unilateral extraction of molars on the 20th postnatal day. After measuring body weight, each experimental animal was anaesthetized with an intraperitoneal injection of sodium pentobarbital ( $0.06 \mathrm{mg} / \mathrm{g}$ body weight). The bilateral inferior alveolar nerves (IAN) in ICR mice were surgically exposed just anterior to the mandibular foramen, and $1 \mathrm{ml}$ of a solution of $5 \%$ HRP-WGA (Toyobo, Osaka, Japan), entrapped in a $1 \%$ hypo-allergenic polyacrylamide gel, was injected by means of a glass micropipette (80-100 mm in diameter) into each nerve bundle. After 24 hours, the deeply anesthetized animals were perfused through the ascending aorta with $200 \mathrm{ml}$ of heparinized saline, which was immediately followed by $300 \mathrm{ml}$ of fixative (1\% paraformaldehyde and $1 \%$ glutaraldehyde in $0.1 \mathrm{M}$ phosphate buffer, $\mathrm{pH} 7.4$ ). The fixative was then flushed out with $200 \mathrm{ml}$ of $10 \%$ sucrose in $0.1 \mathrm{M}$ phosphate buffer, $\mathrm{pH} 7.4$. The brainstem and trigeminal ganglion (TG) were stored overnight in a solution of $30 \%$ sucrose in phosphate buffer $(\mathrm{pH} 7.4)$ at $4^{\circ} \mathrm{C}$. Transverse frozen sections of $50 \mathrm{~mm}$ thickness were serially cut, and the sections were processed in accordance with the tetramethyl-benzidine (TMB) protocol. ${ }^{17}$ The sections were then dried overnight at room temperature (RT) on slides coated with chrome alumgelatin. All treated sections were examined under light microscope (AX70; Olympus, Tokyo, Japan), and the total number of labeled neurons was counted in the mesencephalic trigeminal nucleus (Me5) and TrigeminalGanglion (TG). To avoid double counting, the shape of each labeled cell with a clear nucleus was traced using an aid of camera lucida. All data were analyzed by Student's $t$-test, and differences of $\mathrm{P}<0.05$ were considered to be significant.

\section{Electron microscopic observation of the IAN (Inferior Alveolar Nerve)}

Male mice tooth-extracted on day 20 after birth, were intracardially perfused with a solution of $4 \%$ 
paraformaldehyde and $0.25 \%$ glutaraldehyde in 0.1 $M$ phosphate buffer ( $\mathrm{pH}$ 7.4) under deep anesthesia on day 40 after birth. The IAN were exposed just posterior to the mandibular foramen, and removed. Dissected nerves were immersed in the same fixative for $24 \mathrm{~h}$. Blocks of tissues were then postfixed with $1 \%$ OsO4. These were stained en bloc with $1 \%$ aqueous uranyl acetate, dehydrated with ethanol and propylene oxide, and embedded in Epon 812. Ultra thin sections were stained with uranyl acetate and lead citrate, and examined with an electron microscope (JEM-1010; JEOL, Japan).

\section{Results}

A. Postnatal changes in the mean body weight of tooth-extracted and intact mice

The body weight gradually increased from day 15 to day 360 after birth. No significant difference was detectable between the mean body weights of intact and tooth-extracted mice throughout the days examined. This result indicates that unilateral toothextraction did not cause nutritional handicap in mice examined in the present study and suggests that all data obtained from tooth-extracted mice was independent to the nutritional condition.

\section{B. Labeled Me5 and TG neurons}

Labeled neurons were observed in the Me 5 and TG in both the intact and tooth-extracted mice (Fig.1 and Fig.2). As shown in Figs. 4-A and $-B$, there were significant decreases in the numbers of labeled Me5 and TG neurons in 360-day-old intact mice in comparison with those in 30-day-old intact mice, though we could not detect any significant difference

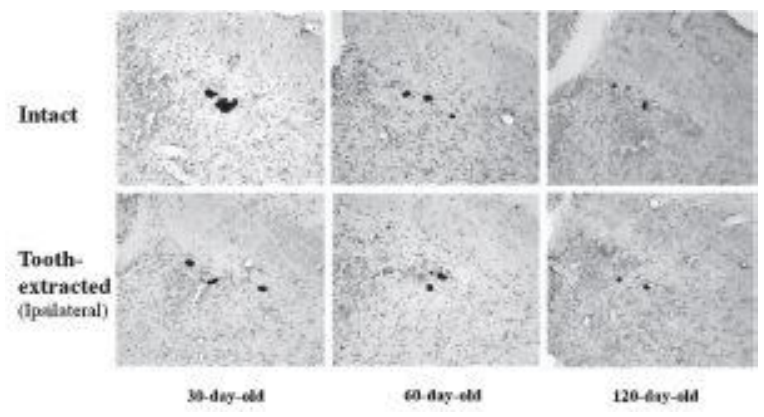

Fig.-1: Labeled neurons in Me5 area of the CNS at the age of 30 day after birth and 60 day after birth respectively, both in intact mice and in the tooth extracted mice.

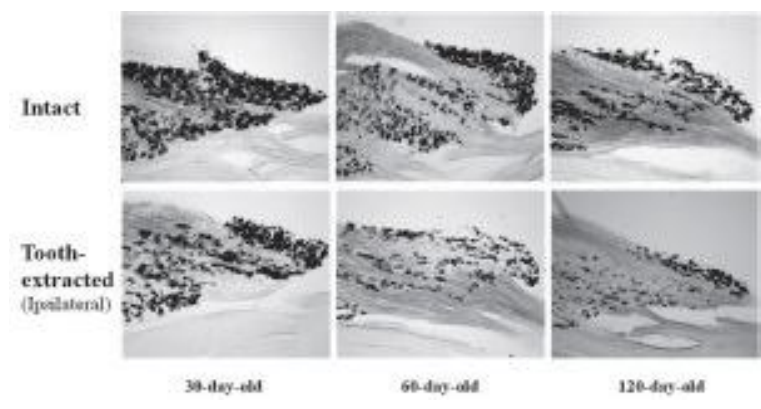

Fig.-2: Labeled neurons in TG at the age of 30 day after birth and 60 day after birth respectively, both in intact mice and in the tooth extracted mice.

in the numbers of labeled Me5 and TG neurons between right and left sides at every time period examined, respectively. Significant differences in the numbers of the labeled Me5 and TG neurons were detected between the tooth-extracted and untreated sides after the 60th postnatal day (Figs. 4-A and -B). Moreover, the numbers of labeled neurons in the Me5 and TG on the contralateral side in the treated mouse was almost same to those in the intact mouse (Figs. 3 and 4).

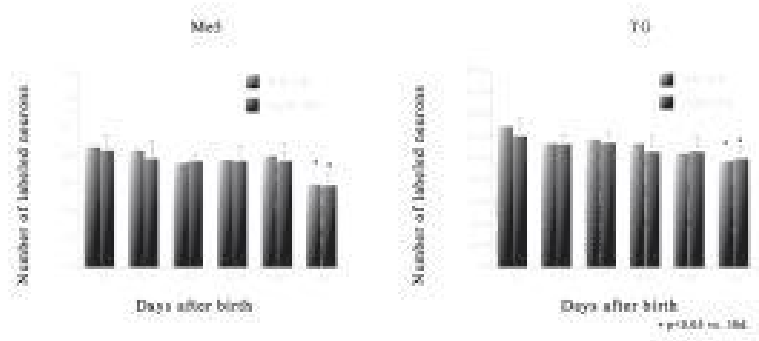

Fig.-3: Number of labeled neurons in the Me5 and $T G$ area of the CNS in the intact (control group) mice. No significant difference was observed between the number of neurons in both the sides.

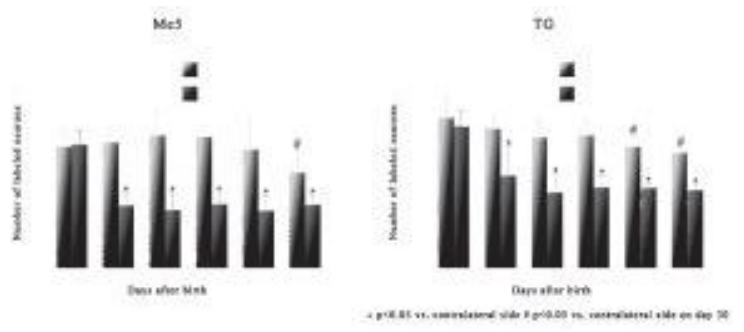

Fig.-4: Number of labeled neurons in the Me5 and TG area of the CNS in the tooth extracted mice. Significant difference was observed between the number of neurons in the teeth extracted side and contralateral side. 
C. Labeled primary afferent terminals in Su5, Pr5,
Sp5O and Sp5I

Fig. 5 shows labeled primary afferent terminals in the supratrigeminal nucleus (Su5), principal sensory trigeminal nucleus (Pr5), spinal trigeminal nucleus oral part (Sp5O), and spinal trigeminal nucleus interpolar part (Sp5I) in the 60-day-old mouse. It was detectable that primary afferent terminals were diminished on the ipsilateral side in the 60-day-old teeth-extracted mouse, as compared to those on the contralateral side. Any significant difference was undetectable in the labeled terminals between both sides in intact mice and the contralateral side in tooth-extracted mice. Significant reductions were also observed in the terminals of the 360-day-old mouse, as compared with those of the 30-day-old mouse (photographic image not shown).

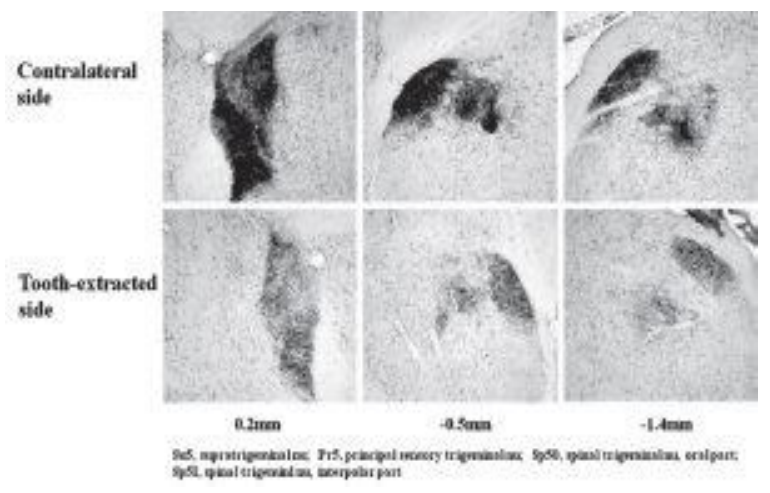

Fig.-5: Labeled primary afferent terminals in the supra-trigeminal nucleus (Su5), principal sensory trigeminal nucleus (Pr5), spinal trigeminal nucleus oral part (Sp5O) and spinal trigeminal nucleus interpolar part (SP5I) in the 60 day old mouse. It was detectable that primary afferent terminals were diminished on the ipsilateral side in the 60 day old tooth extracted mice as compared to those on the contralateral side.

\section{Electron microscopy of the IAN}

Many degenerated nerve fibers were observed on the treated side in the IAN in the 40-day-old toothextracted mouse. Typical degenerative changes were detected in the myelin sheaths and axons in the IAN of the tooth extracted side (Fig.6-B), although any degenerative nerve fiber was not found on the contralateral side (Fig.6-A).
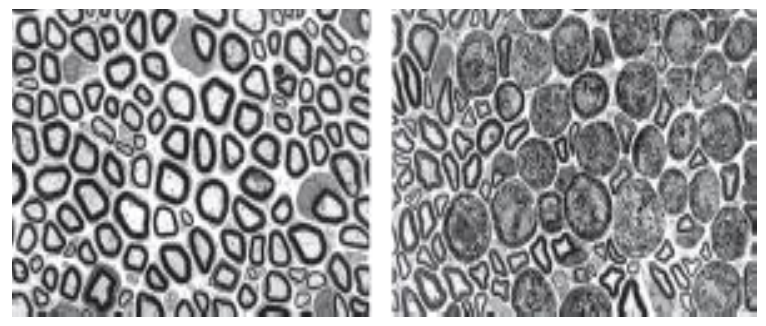

Fig.-6: Electron microscopic view of the IAN in the 40 day old tooth extracted mouse. Typical degenerative changes are seen in the myelin sheaths and axons in the IAN of the tooth extracted side (7-B) although any degenerative nerve fibers was found on the contralateral side (7-A).

\section{Discussion}

There are many studies focused on the system regulating the jaw movement. $1,2,3,4,6,8,9$, $10,11,15,16,18,19$ It had been suggested that the sensory input from the oro-facial sensory receptors play an important role in controlling the formation of the masticatory system during certain stage of growth in animals. ${ }^{3}$ Maeda et al. reported that the masseter muscle was atrophied in the mice with extraction of unilateral molars and in the osteopetrotic (op/op) mouse with no eruption of teeth and less-developed periodontal ligaments. ${ }^{20,21,22}$ On the other hand, there is significant decrease in the number of jaw closing motoneurons innervating the masseter muscle in 3- and 5-month-old op/op mice. ${ }^{15}$ Masticatory sensory neurons were also less in number in the 3-month-old op/op mouse, as compared with those in the normal mouse. ${ }^{16}$ All these observations in the mutant op/op mouse suggest that the sensory input from the PMRs may play an important role in the postnatal development of the masticatory system and maintaining the homeostatic condition in the system regulating the jaw movement. Now, the question arises whether such suggestion based on results from the mutant op/op mouse, is generally applied to the normal animal in which tooth is lost during certain stage of the development.

Mechanoreceptors in the periodontal ligaments are innervated by $\mathrm{Me} 5$ and TG neurons ${ }^{23}$ and the central axons of the Me5 neurons project mainly to the Su5 and descend Probst's tract. ${ }^{24}$ The Su5 contains not only inhibitory neurons but also excitatory 
neurons with respect to the jaw closing motoneurons. ${ }^{1,2,4,25}$ In the present study, the serious degeneration of the IAN in the toothextracted mouse was certainly detected prior to the significant reduction in the neuron numbers of the Me5 and TG, and the primary afferents in the trigeminal sensory nuclear complex (TSNC) in the tooth-extracted mice. Therefore, it is strongly suggested that the disorder of the jaw closing activity is surely caused in the tooth-extracted mice. On the other hand, the primary afferents originating from the TG form the terminals to the TSNC ${ }^{26}$ and project to the Mo5 via Sp5O. ${ }^{27}$ The dorsomedial part of the $\mathrm{Sp5O}$ is a region that receives primary afferents of intra-oral structure, and the neurons in the Sp5O have widely spreading dendrites and axons that project to the Mo5. ${ }^{7,24}$ Moreover, the neurons in the $\mathrm{Sp5O}$ receiving the periodontal Me5 input are related to the formation of a disynaptic or multisynaptic segmental reflex pathway. ${ }^{8}$ The rostrodorso-medial part of the Sp5O contains interneurons to the jaw closing and opening motor neurons in the Mo5 ${ }^{11}$. Suemune and Maeda had detected a significant decrease in the number of TG neurons and observed the severe reduction of labeled terminals in the Sp5O in the op/op mouse. Furthermore, they found that the decrease in the number of TG neurons was greater in the op/op mouse than that in the normal mouse fed a granulated diet ${ }^{16}$ and inferred that the inevitable decrease in the sensory input to the Sp5O via TG from periodontal sensory endings might cause the reduction of the $\mathrm{Sp} 5 \mathrm{O}$ terminals in the op/op mouse. In the present experimental model, a very similar result was also obtained in the labeled primary afferents in the TSNC. Therefore, it can be confirmed that the severe disorder of the system regulating the mastication is also induced by the tooth loss even in the normal animal.

\section{Conclusion}

The present study thus suggests that the decrease in the sensory input due to inevitable degeneration of the PMRs, resulted from tooth loss, causes the disorder of the development and maintenance of the masticatory system even in the normal animal. Again, aging is also a factor that results into disorder of the masticatory system, though further study is needed to reveal the status of the PMRs' in old aged mice. In addition, the present study provides a stable and well-characterized model for the examinations of the regulation of neuronal survival and death in the masticatory sensory neurons following tooth loss.

\section{References}

1. Kidokoro Y., Kubota K., Shuto S. and Sumino R.: Reflex organaization of masticatory muscles in the cat. J. Neurophysiol. 1968a. 31, 695-708.

2. Kidokoro Y., Kubota K., Shuto S. and Sumino R.: Possible interneurons responsible for reflex inhibition of motoneurons of jaw-closing muscles from the inferior dental nerve. $J$. Neurophysiol. 1968b. 31: 709-16.

3. Kubota K.: Role of sensory input in formation of masticatory system. In: Kubota K. (Ed.) Mechanobiological Research on the Masticatory System. VEB Verlage, Berlin . 1989. 62.

4. Goldberg L.J.: Masseter muscle excitation induced by stimulation of periodontal and gingival receptors in man. Brain Res. 1971; 32, 369-81.

5. Limwongse V. and De Santis M.: Cell body locations and axonal pathways of neurons innervating muscles of mastication in the rat. m. J. Anat. 1977; 149: 477-88.

6. Linden R.W. and Scott B. J.: The effect of tooth extraction on periodontal ligament mechanoreceptors represented in the mesencephalic nucleus of the cat. Arch. Oral Biol. 1989; 34: 937-41.

7. Shigenaga Y., Okamoto T., Nishimori T., Suemune S., Nasution I.D., Chen, I.C., Tsuru K., Yoshida A., Tabuch, K., Hosoi M., and Tsuru $H_{\text {.: }}$ Oral and facial representation in the trigeminal principal and rostral spinal nuclei of the cat. J. Com. Neurol. 1986; 244: 1-18.

8. Shigenaga Y., Doe K., Suemune S., Mitsuhiro Y., Tsuru K., Otani K., Shirana Y., Hosoi M., Yoshida A. and Kagawa K.: Physiological and morphological characteristics of periodontal mesencephalic trigeminal neurons in the catintra-axonal staining with HRP. Brain Res. 1989; 505, 91-110.

9. Shigenaga Y., Mitsuhiro Y., Shirana Y. and Tsuru H.: Two types of jaw-muscle spindle afferents in the cat as demonstrated by intra- 
axonal staining with HRP. Brain Res. 1990; 514: 219-37.

10. Yoshida A., Mukai Y., Moritani M., Nagase Y., Hirose Y., Homma S., Fukami H., Takagi K., Matsuya T. and Shigenaga Y. Physiologic and morphologic properties of motoneurons and spindle afferents innervating the temporal muscle in the cat. J. Comp. Neurol. 1999; 406: 29-50.

11. Yoshida A., Fukami H., Nagase Y., Appenteng K., Homma S., Zhang L-F., Bae Y.C. and Shigenaga Y. Quantitative analysis contacts made between functionally identified oralis neurons and trigeminal motoneurons in cats. J. Neurosci. Let. 2001; 21: 6298-307.

12. Momose I., Nishikawa J., Watanabe Y., Sasaki Y., Senda M., Kubota K., Sato Y., Funakosh, M. and Minaskuchi S.: Effect of mastication on regional cerebral blood flow in human examined by positron-emission tomography with ${ }^{15} \mathrm{O}$-labeled water and magnetic resonance imaging. Arch. Oral Biol. 1977; 42: 57-61.

13. Simpson, $\mathrm{H}$. E. The healing of extraction wound ; Br. Dent. J. 1968; 126: 550-57.

14. Kato, T., Usami, T., Noda, Y., Hasegawa, M., Ueda, M. and Nabeshima, T.: The effect of the loss of molar teeth on spatial memory and acetylcholine release from the parietal cortex in aged rats. Behav. Brain Res. 1997; 83: 239-42.

15. Suemune, S., Kawata, K. and Maeda, N.: Reduction of masticatory motoneurons in the trigeminal motor nucleus of the toothless (op/ op) mouse. Biomed. Res. 1994; 15: 45-49.

16. Suemune, S. and Maeda, N.: Reduction in the number of motor and sensory neurons that are involved in the jaw-opening reflex in the toothless (op/op) mouse. Biomed. Res. 1994; 15: 63-69.

17. Mesulam MM. Tetramethyl benzidine for horseradish peroxidase neurohistochemistry: a non-carcinogenic blue reaction-product with superior sensitivity for visualizing neural afferents and efferents. J. Histochem. Cytochem. 1978; 26: 106-17.

18. Nakamura Y., Nagashima H. and Mori S.: Bilateral effects of the afferent impulses from the masseteric muscle on the trigeminal motoneuron of the cat. Brain Res. 1973; 57: 15-27.

19. Jacquin M. F., Rhoades R. W., Enfiejian H. L. and Egger M. D.: Organization and morphology of masticatory neurons in the rat: A retrograde HRP study. J. Comp. Neurol. 1983; 218: 23956.

20. Maeda N., Kawasaki T., Osawa K., Yamamoto Y., Sumida H., Masuda T. and Kumegawa M.: Effects of long-term intake of a fine-grained diet on the mouse masseter muscle. Acta Anat. 1987; 128: 325-33.

21. Maeda N., Kawata T., Yoshiko Y., Hosoi M., Suemune S., Okada N. and Tanne K.: Postnatal changes in the masseter muscle of the toothless (op/op) mouse with lessdeveloped periodontal ligaments. Biomed. Res. 1994; 15: 255-61.

22. Maeda N., Suwa T., Ichikawa M., Masuda T. and Kumegawa M.: Effects of easily chewable diet and unilateral extraction of upper molars on the masseter muscles in developing mice. Acta Anat. 1990; 137: 19-24.

23. Byers M.R. and Dongs W.K.: Comparison of trigeminal receptor location and structure in the periodontal ligament of different types of teeth from the rat, cat, and monkey. J. Comp. Neurol. 1989; 279: 117-27.

24. Shigenaga Y., Sera M., Nishimori T., Suemune S., Nishimura M., Yoshida A. and Tsuru K. : The central projection of masticatory afferent fibers to the trigeminal sensory nuclear complex and upper cervical spinal cord. J. Comp. Neurol. 1988; 268; 489-07.

25. Jerge C.R.: The function of the nucleus supratrigeminalis. J Neurophysiol. 1963; 26: 393-402.

26. Tsuru k., Otani K., Kajiyama K., Suemune S., Shigenaga Y.: Central terminations of periodontal mechanoreceptive and tooth pulp afferents in the trigeminal principal and oral muclei of the cat. Brain Res. 1989; 485: 2961.

27. Sumino R.: Central neural pathways involved in the jaw-opening reflex in the cat. In Kawamura Y. (Ed.) Dubner, R Oral-facial Sensory and Motor Mechanism. AppletonCentury-Crofts, New York, 1971; 315-31. 